\title{
Report from a joint research project for the use of retractable membrane structures in South Korea
}

\author{
Kyung-Ju Hwang ${ }^{*}$, Alexander Hub ${ }^{\dagger}$ \\ * School of Architecture at University of Seoul \\ 163 Seoulsiripdae-ro, Dongdaemun-gu, Seoul 130-743 Korea \\ e-mail: kj.hwang@uos.ac.kr, web page: http://archi.uos.ac.kr \\ ${ }^{\dagger}$ Alfred Rein Ingenieure $\mathrm{GmbH}$ \\ Fuchseckstraße 7, 70188 Stuttgart, Germany \\ e-mail: mail@ar-ingenieure.com, web page: http://ar-ingenieure.com
}

\begin{abstract}
Since the 2017 conference, a Korean collaborative research project has been reported, which aims to develop the principles for an economical and lightweight convertible roofing solution. Various research and business working groups have been developing solutions to these issues over the past four years. In this context, a far-reaching monitoring concept should also enable the validation of the results through the monitoring and analysis of the roof system operation.
\end{abstract}

As a prototype test object, the access to a subway station in Seoul is covered with a $1,700 \mathrm{~m} 2$ convertible membrane roof. The structure, designed by MIM Architects, can be driven up with a total of 104 supporting points on 10 cable axes arranged in a fan shape and 2 rail axes. A novel feature is that the construction site does not allow for a symmetrical structure, so the design concepts for accommodating non-uniform loads can be effectively tested on it. The TestBed is scheduled for completion at its Munjung Culture Valley site in the fall of 2021, when it will represent the largest convertible membrane roofing system in South Korea.

Highly qualified Korean companies from the ropeway and roller coaster construction sector are involved in the realization of the construction project for the planning of the drive concepts. The research concept provides for a close practice-oriented interlinking of research and industry. In working groups, solutions were developed for individual structural components, the function of which was tested in real component tests. In this project phase, AR Ingenieure continued to act in an advisory capacity. Numerical membrane folding simulations were carried out to investigate the interaction of the geometrically different drive axles under different driving conditions. The results were used to formulate a recommendation for the control concept.

The focus of the consulting services is particularly on the development of the roof's travel components. For a roof of this size, a combined mechanism is used for the first time, which carries out both the driving process of the roof membrane and the final tensioning process with the same drive. An autonomously locking and releasing anchoring mechanism was designed for anchoring the membrane when in use in order to reliably transfer the high static forces of the roof into the pressure ring.

The talk will present exemplary results from the research groups, as well as report on the completion of the TestBed in Seoul. 